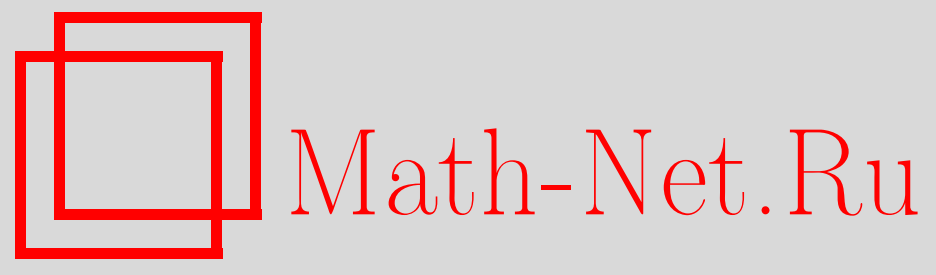

А. Ю. Заиграев, А. В. Нагаев, Абелевы теоремы, граничные свойства сопряженных распределений и большие уклонения сумм независимых случайных векторов, Теория вероятн. и ее примен., 2003, том 48, выпуск 4, 701-719

DOI: https://doi.org/10.4213/tvp252

Использование Общероссийского математического портала MathNet.Ru подразумевает, что вы прочитали и согласны с пользовательским соглашением

http://www . mathnet.ru/rus/agreement

Параметры загрузки:

IP : 3.80 .253 .173

26 апреля 2023 г., 17:25:03

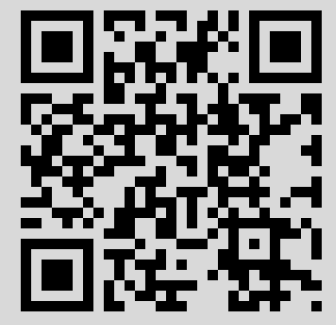




\section{АБЕЛЕВЫ ТЕОРЕМЫ, ГРАНИЧНЫЕ СВОЙСТВА СОПРЯЖЕННЫХ РАСПРЕДЕЛЕНИЙ И БОЛЬШИЕ УКЛОНЕНИЯ СУММ НЕЗАВИСИМЫХ СЛУЧАЙНЫХ ВЕКТОРОВ ${ }^{1)}$}

Рассматривается класс многомерных абсолютно непрерывных распределений. Каждое распределение имеет конечную в некотором ограниченном выпуклом множестве $S$ производящую функцию, порождаюшую семейство так называемых сопряженных распределений. В центре внимания находятся предельные распределения для этого семейства, когда параметр сопряжения стремится к границе $S$. Как и в одномерном случае, каждое такое предельное распределение может быть получено как следствие теоремы абелева типа. Полученные результаты используются для установления локальной предельной теоремы для больших уклонений произвольно высокого порядка.

Ключевые слова и фразы: гамма-подобное распределение, локальная предельная теорема, опорная функция, правильное изменение, сверхбольшие уклонения, условие Крамера, функция уклонений.

1. Введение. Пусть случайные векторы $\xi, \xi^{(1)}, \ldots, \xi^{(n)}, \ldots$ со значениями в $\mathbf{R}^{d}, d \geqslant 1$, независимы и имеют общее сушественно $d$-мерное распределение $\mathbf{P}_{0}$. Образуем суммы $S_{n}=\xi^{(1)}+\cdots+\xi^{(n)}, n=1,2, \ldots$.

Если $\mathbf{E}|\xi|^{2}<\infty$, то, согласно центральной предельной теореме, для любого $A$, являющегося множеством непрерывности $d$-мерной меры Лебега, при $n \rightarrow \infty$

$$
\mathbf{P}\left\{n^{-1 / 2}\left(S_{n}-n a\right) \in A\right\} \rightarrow \int_{A} \varphi_{B}(u) d u,
$$

где $a=\mathbf{E} \xi$, а $\varphi_{B}(u)$ - плотность случайного вектора, имеюшего $d$ мерное нормальное распределение с нулевым вектором математических ожиданий и ковариационной матрицей $B=\mathbf{E}(\xi-a)(\xi-a)^{T}$.

Если множество $A$ изменяется вместе с $n$ таким образом, что $\inf _{x \in A}|x| \rightarrow \infty$, то возникает так называемая проблема больших уклонений, состоящая в нахождении асимптотики стремящейся к нулю вероятности $\mathbf{P}\left\{n^{-1 / 2}\left(S_{n}-n a\right) \in A\right\}$. Нам будет далее удобно называть вероятностью больших уклонений $\mathbf{P}\left\{S_{n}-n a \in A\right\}$ при $r_{n}(A)=$

\footnotetext{
* Университет Николая Коперника, Торунь, Польша.
} 
$n^{-1 / 2} \inf _{x \in A}|x| \rightarrow \infty$. В случае $r_{n}(A)=O\left(n^{1 / 2}\right)$ будем говорить о больших уклонениях порядка $O\left(n^{1 / 2}\right)$. При $n^{-1 / 2} r_{n}(A) \rightarrow \infty$ можно говорить о сверхбольиих уклонениях. В отличие от [6]-[8], нас интересует точная асимптотика вероятности больших уклонений, а не грубая, иначе логарифмическая, асимптотика.

В контексте точной асимптотики к настоящему времени наиболее полно изучен случай $d=1$. Желающему получить представление о нем можно порекомендовать обзор [21], а также работы [14], [20], [24], [26], [28], [29].

Впервые достаточно общие результаты по проблеме больших уклонений в многомерном случае были получены в [3] в предположении, что выполнено так называемое условие Крамера

$$
f(s)=\mathbf{E} e^{\langle s, \xi\rangle}=\int_{\mathbf{R}^{d}} e^{\langle s, x\rangle} \mathbf{P}_{0}(d x)<\infty, \quad s \in S,
$$

где $S \subset \mathbf{R}^{d}$ - существенно $d$-мерное множество, а $\langle\cdot, \cdot\rangle$ обозначает скалярное произведение в $\mathbf{R}^{d}$. Там же были изучены основные свойства функции $f(s)$ и функиии уклонений

$$
\rho(x)=\inf _{s \in S} f(s) e^{-\langle s, x\rangle}, \quad x \in \mathbf{R}^{d},
$$

играюшей важную роль в изучении вероятностей больших уклонений. Стоит отметить, что многие авторы функцией уклонений называют $-\ln \rho(x)$ (см., например, [4], [6]-[8]).

При выполнении условия (1.1) вероятности больших уклонений изучаются с помошью так называемых сопряженных распределений. Для исходного распределения $\mathbf{P}_{0}$ сопряженное распределение $\mathbf{P}_{s}, s \in S$, определяется с помошью соотношения

$$
\mathbf{P}_{s}(A)=(f(s))^{-1} \int_{A} e^{\langle s, x\rangle} \mathbf{P}_{0}(d x) .
$$

Обозначим через $\gamma(s)$ и $B(s)$ соответственно градиент и гессиан $\ln f(s)$. Легко видеть, что $\gamma(s)$ является вектором средних, а $B(s)-$ ковариационной матрицей сопряженного распределения $\mathbf{P}_{s}$. Поскольку исходное распределение существенно $d$-мерно, то $\operatorname{det} B(s)>0$ и, следовательно, вектор-функция $\gamma(s)$ взаимно однозначно отображает $S$, область сушествования $f(s)$, в некоторую область $X \subset \mathbf{R}^{d}$. Обозначим через $s(x)$ функцию, обратную к $\gamma(s)$.

В упомянутой работе [3] были получены локальная и интегральные предельные теоремы для больших уклонений. Любопытно, что основные результаты той работы были почти через 20 лет воспроизведены в [22] без какого бы то ни было упоминания о ней.

Сформулируем одно следствие из локальной теоремы, доказанной в [3]. Пусть $F$ - произвольное замкнутое ограниченное подмножество множества внутренних точек $S$, а $\gamma(F)$ - его образ при отображении $\gamma$. 
Предложение 1.1. Предположим, что при $n \geqslant n_{0} \geqslant 1$ существует и ограничена $p_{n}(x)$ - плотность распределения суммь $S_{n}$. Если выполняется также условие Крамера (1.1), то при $n \rightarrow \infty$

$$
\sup _{x \in \gamma(F)}\left|\frac{p_{n}(n x)}{\psi_{n}(x) \rho^{n}(x)}-1\right|=o(1)
$$

где $\psi_{n}(x)=(2 \pi n)^{-d / 2}[\operatorname{det} B(s(x))]^{-1 / 2}$.

В существенно более поздней работе [4] были продолжены исследования свойств функции уклонений, а также доказаны более сильные формы интегральной и локальной теорем о больших уклонениях, в которых утверждение предложения 1.1 выполняется равномерно в некотором классе исходных расцределений.

Следует отметить, что в упомянутых работах так же, как и в [1], [2], $[13],[15],[23],[27],[31],[32]$ изучался случай больших уклонений порядка $O\left(n^{1 / 2}\right)$.

Возникает вопрос: при каких дополнительных условиях можно снять ограничения на порядок уклонений? Точнее, каким должно быть исходное распределение, чтобы выполнялось соотношение

$$
\sup _{x \in \mathbf{R}^{d}}\left|\frac{p_{n}(n x)}{\psi_{n}(x) \rho^{n}(x)}-1\right|=o(1) ?
$$

Основной целью данной работы является попытка найти хотя бы частичный ответ на этот вопрос. При этом мы рассматриваем случай, когда множество $S$ ограничено, a $\lim _{s \rightarrow \partial S} f(s)=\infty$. В этой связи уместно вспомнить работы [17], [18], посвященные большим уклонениям на полупрямой $\mathbf{R}_{+}^{1}$. При $d=1$ ограниченность $S=\left(s_{-}, s_{+}\right)$означает, в частности, что $0 \leqslant s_{+}<\infty$. Из упомянутых работ следует, что при $0<s_{+}<\infty$ случаи $f\left(s_{+}\right)=\infty$ (см. [17]) и $f\left(s_{+}\right)<\infty$ (см. [18]) отвечают совершенно разному поведению вероятностей сверхбольших уклонений. Таким образом, в данной работе нас интересует многомерный аналог случая, рассмотренного в [17].

Как обычно, при переходе к высшим размерностям возникает целый ряд вопросов. К числу таковых относятся, например, вопросы о том, как связана форма области существования производяшей функции $f(s)$ со свойствами исходного распределения, как ведет себя $f(s)$ при $s \rightarrow \partial S$, что представляет собой область, в которую $\gamma(s)$ отображает $S$, и т.п. Ответы на эти вопросы, имеющие и самостоятельный интерес с точки зрения теории асимптотических оценок, были даны в работе [19]. Содержащиеся в ней результаты играют существенную роль в приводимых ниже рассмотрениях.

Несколько слов о построении данной работы. Во втором разделе описывается один класс абсолютно непрерывных распределений, для ко- 
торых справедливо соотношение (1.2). Третий раздел посвящен предельным теоремам для сопряженных распределений. Доказательство основного результата (1.2) и его статистическая интерпретация даны в заключительном четвертом разделе.

Авторы весьма признательны рецензенту за ценные замечания.

2. Формулировка основного результата. Введем следующий класс абсолютно непрерывных распределений, удовлетворяющих условию Крамера.

О п р е д е л ен и е 2.1. Для ненулевого вектора $x \in \mathbf{R}^{d}$ определим $e_{x}=|x|^{-1} x \in S^{d-1}$. Будем говорить, что бункиия $a(x)$ принадлежит классу $\mathscr{A}$, если

1) а

2) $a(x)$ однородна в том смисле, ито $a(x)=|x| a\left(e_{x}\right)$ для всех $x \neq 0$;

3) $0<\min _{e \in S^{d-1}} a(e) \leqslant \max _{e \in S^{d-1}} a(e)<\infty$.

Каждая функция $a(x) \in \mathscr{A}$ является опорной для некоторого ограниченного выпуклого множества, имеющего начало координат своей внутренней точкой (см., например, [30, гл. 3, § 13]).

Следуюшее определение правильно изменяющейся функции в $\mathbf{R}^{d}$ стоит сравнить с определением, приведенным в [25, гл. 5.4.2].

О п р е д е л е н и е 2.2. Пусть неотрицательная функиия $\lambda($ e) непрерьвна на единичной сфере $S^{d-1}$. Будем говорить, что функиия $b(x), x \in \mathbf{R}^{d},(\beta, \lambda)$-правильно изменяется, если при $|x| \rightarrow \infty$

$$
\sup _{e_{x} \in E_{\lambda}}\left|\frac{b(x)}{r_{\beta}(|x|)}-\lambda\left(e_{x}\right)\right|=o(1),
$$

где $r_{\beta}(t)$ правильно изменяется при $t \rightarrow \infty$ с показателем $\beta$, а $E_{\lambda}=\left\{e \in S^{d-1}: \lambda(e)>0\right\}$. Eсли $E_{\lambda}=S^{d-1}$, то говорим просто о $\beta$ правильном изменении.

Условимся обозначать через $\chi_{d-1}$ нормированную меру Хаара на $S^{d-1}$.

Рассмотрим плотность вида

$$
p(x)=b(x) e^{-a(x)}, \quad x \in \mathbf{R}^{d},
$$

где $a(x) \in \mathscr{A}$, а $b(x)-\beta$-правильно изменяется при $|x| \rightarrow \infty$.

В частном случае, когда

$$
b(x)=\frac{q\left(e_{x}\right)|x|^{\alpha-1}}{\Gamma(\alpha+d-1)}, \quad \alpha+d-1>0,
$$

где $\int_{S^{d-1}} q(e)(a(e))^{-\alpha-d+1} \chi_{d-1}(d e)=1$, получаем плотность

$$
p(x)=g_{\alpha}(x ; a, q)=\frac{q\left(e_{x}\right)|x|^{\alpha-1} e^{-a(x)}}{\Gamma(\alpha+d-1)},
$$


которую можно с полным основанием назвать $d$-мерной гаммаплотностью.

В отличие от одномерного случая параметрического семейства гамма-распределений с общей плотностью вида

$$
p(x)=g_{\alpha}(x ; \lambda)=\frac{\lambda^{\alpha} x^{\alpha-1} e^{-\lambda x}}{\Gamma(\alpha)}, \quad x>0,
$$

где $\lambda>0, \alpha>0$, в многомерном случае плотности вида (2.2) образуют полупараметрическое семейство.

Гамма-распределение в $\mathbf{R}^{1}$, подобно нормальному, обладает свойством воспроизводимости при переходе к сопряженным распределениям. Точнее, пусть $p_{s}(x)$ - плотность, отвечающая сопряженному распределению $\mathbf{P}_{s}$. Легко понять, что сопряженная к (2.3) плотность имеет вид

$$
p_{s}(x)=g_{\alpha}^{(s)}(x ; \lambda)=g_{\alpha}(x ; \lambda-s), \quad s<\lambda .
$$

Аналогично этому при $d>1$ и $p(x)=g_{\alpha}(x ; a, q)$ производящая функция имеет вид

и, следовательно,

$$
f(s)=\int_{S^{d-1}} q(e)(a(e)-\langle s, e\rangle)^{-\alpha-d+1} \chi_{d-1}(d e),
$$

$$
p_{s}(x)=g_{\alpha}^{(s)}(x ; a, q)=g_{\alpha}\left(x ; a_{s}, q_{s}\right)
$$

где

$$
a_{s}(\varepsilon)=a(\varepsilon)-\langle s, \varepsilon\rangle, \quad q_{s}(\varepsilon)=\frac{q(\varepsilon)}{\int_{S^{d-1}} q(e)(a(e)-\langle s, e\rangle)^{-\alpha-d+1} \chi_{d-1}(d e)} .
$$

Таким образом, класс распределений (2.2) замкнут относительно преобразования Крамера - перехода к сопряженному распределению.

Из определения 2.2 и формулы (2.2) нетрудно понять, что плотность вида $(2.1)$, где $b(x)-(\alpha-1)$-правильно изменяется с $\alpha>$ $1-d$, при $|x| \rightarrow \infty$ с точностью до медленно изменяющейся функции ведет себя как введенная нами $d$-мерная гамма-плотность с $q(e)=\lambda(e) \Gamma(\alpha+d-1)$. Поэтому такие плотности мы называем асимптотически гамма-подобными или просто гамма-подобными.

В дальнейшем всюду мы рассматриваем только такие плотности.

Легко понять, что класс гамма-подобных плотностей является достаточно богатым. Помимо $d$-мерных гамма-плотностей он содержит, например, эллипсоидальные плотности вида

$$
p(x)=K_{d}\left(x^{T} A x\right)^{(\alpha-1) / 2} \exp \left(-\left(x^{T} A x\right)^{1 / 2}\right), \quad K_{d}>0
$$

где $A$ - положительно определенная матрица.

Если $a(x) \in \mathscr{A}$, а $S$ - соответствующее ей, как опорной функции, ограниченное выпуклое множество, то, согласно определению,

$$
a(x)=\sup _{y \in S}\langle y, x\rangle=|x| \sup _{y \in S}\left\langle y, e_{x}\right\rangle=|x| a\left(e_{x}\right), \quad x \in \mathbf{R}^{d} .
$$


Рассмотрим функцию

$$
k(y)=\inf \{\lambda \geqslant 0: y \in \lambda S\}, \quad y \in \mathbf{R}^{d} .
$$

Известно (см., например, $[30$, гл. $3, \S 15])$, что

$$
k(y)=\sup _{x \neq 0} \frac{\langle y, x\rangle}{a(x)}=|y| \sup _{e \in S^{d-1}} \frac{\left\langle e_{y}, e\right\rangle}{a(e)} .
$$

Положим $h(e)=1 / k(e)$. Тогда множество внутренних точек $S$ допускает представление

$$
\operatorname{int} S=\{s: s=t e, 0 \leqslant t<h(e)\} .
$$

Заметим, что функции $a(e)$ и $k(y)$ иногда называют функциями Минковского.

В работе [19] была установлена форма области сушествования производящей функции $f(s)=\int_{\mathbf{R}^{d}} e^{\langle s, x\rangle} p(x) d x$. Оказывается, если $p(x)$ имеет представление $(2.1)$, где $a(x) \in \mathscr{A}$, а $b(x)-\beta$-правильно изменяется при $|x| \rightarrow \infty$, то производящая функция $f(s)$ ограничена в $\operatorname{int} S$, где $S$ - ограниченное выпуклое множество, содержащее начало координат, для которого $a(x)$ является опорной функцией. И наоборот, для любого ограниченного выпуклого множества $S$ такого, что $0 \in \operatorname{int} S$, существует плотность вида (2.1), имеющая $S$ областью сушествования $f(s)$.

Заметим, что при $\beta \geqslant-d$ область существования $f(s)$ открыта, поскольку $f(t e) \rightarrow \infty$ при $t \rightarrow h(e)$. Если же $\beta<-d$, то $f(h(e) e)<\infty$, т.е. $f(s)$ конечна в замкнутой области $S=\{s: s=t e, 0 \leqslant t \leqslant h(e)\}$. Как уже отмечалось во введении, нас интересует случай открытого множества существования $f(s)$.

Перейдем теперь к формулировке основного результата нашей работы. Обозначим $\Delta_{e}(\varepsilon)=a(\varepsilon)-h(e)\langle e, \varepsilon\rangle$, где $h(e)$ имеет тот же смысл, что и в (2.4). Нам потребуются следующие предположения.

(A) Для любого направления $е \in S^{d-1}$ множество

$$
\arg \min _{\varepsilon \in S^{d-1}:\langle e, \varepsilon\rangle>0}\left[a(\varepsilon)\langle e, \varepsilon\rangle^{-1}\right]
$$

содержит единственную точку $\varepsilon^{\prime}=\varepsilon^{\prime}(e)$.

(В) Для любого направления е $\in S^{d-1}$ функиия $\Delta_{e}(\varepsilon)$ в окрестности точки $\varepsilon^{\prime}($ e) допускает представление

$$
\Delta_{e}(\varepsilon)=2^{-1}\left(\varepsilon-\varepsilon^{\prime}(e)\right)^{T} \Lambda_{e}\left(\varepsilon-\varepsilon^{\prime}(e)\right)+w_{e}(\varepsilon),
$$

где матрица $\Lambda_{e}$ неотрииательно определена и имеет ранг $d-1$, а $\left|w_{e}(\varepsilon)\right|=o\left(\left|\varepsilon-\varepsilon^{\prime}(e)\right|^{2}\right)$. При этом матрица $\Lambda_{e}$ непрерывна по е $\in S^{d-1}$, $\Lambda_{e} \varepsilon^{\prime}(e)=0 u$

$$
0<\min _{e \in S^{d-1}} \min _{j} \lambda_{j}(e) \leqslant \max _{e \in S^{d-1}} \max _{j} \lambda_{j}(e)<\infty, \quad j=1, \ldots, d-1,
$$

где $\left\{\lambda_{j}(e)\right\}_{j=1}^{d-1}$ - ненулевье собственные числа $\Lambda_{e}, a$

$$
\sup _{e \in S^{d-1}} \sup _{\left(\varepsilon:\left|\varepsilon-\varepsilon^{\prime}(e)\right| \leqslant t\right)}\left|w_{e}(\varepsilon)\right| \leqslant w(t)=o\left(t^{2}\right), \quad t \rightarrow 0 .
$$


(C) Для $\varepsilon \in S^{d-1}$ и всех достаточно мальих $\delta>0$

$$
\min _{e \in S^{d-1}} \min _{\left(\varepsilon \in S^{d-1}:\left|\varepsilon-\varepsilon^{\prime}(e)\right|>\delta\right)} \Delta_{e}(\varepsilon)=d(\delta)>0 .
$$

Теорема 2.1. Если гамма-подобная плотность удовлетворяет условиям $(A),(B)$ и $(C)$, то справедливо (1.2).

Прежде чем приступить к доказательству сформулированной теоремы, уместно заметить следующее.

Из условия (A), в частности, следует, что в каждой точке $\varepsilon^{\prime}(e)$ существует только одна опорная гиперплоскость к $S$, т.е. граница выпуклой области $S$ не имеет угловых точек. Более того, кривизна границы равномерно положительна и ограничена. Это справедливо, например, в случае, когда $S$ - эллипсоид, но не имеет места в случае, когда, например, $S-$ квадрат.

П р и м е р 2.1. Пусть

$$
p(x)=b(x) e^{-\left(x^{T} B x\right)^{1 / 2}}, \quad x \in \mathbf{R}^{d},
$$

где функция $b(x) \beta$-правильно изменяется при $|x| \rightarrow \infty$ с $\beta \geqslant-d$, а $B-$ положительно определенная матрица.

Нетрудно видеть, что в этих условиях

$$
a(e)=\left(e^{T} B e\right)^{1 / 2}, \quad h(e)=\left(e^{T} B^{-1} e\right)^{-1 / 2}, \quad e \in S^{d-1},
$$

т.е. область $S$ представляет собой эллипсоид

$$
S=\left\{s \in \mathbf{R}^{d}: s=t e, 0 \leqslant t<\left(e^{T} B^{-1} e\right)^{-1 / 2}\right\} .
$$

В следующем примере граница области $S$ имеет угловые точки.

П р и м е р 2.2. Пусть $d=2$, а

$$
p(x)=b(x) e^{-\left|x_{1}\right|-\left|x_{2}\right|}, \quad x \in \mathbf{R}^{2},
$$

где функция $b(x) \beta$-правильно изменяется при $|x| \rightarrow \infty$ с $\beta \geqslant-2$.

Нетрудно видеть, что в этих условиях

$$
a(e)=\left|e_{1}\right|+\left|e_{2}\right|, \quad h(e)=\min \left\{\left|e_{1}\right|^{-1},\left|e_{2}\right|^{-1}\right\}, \quad e \in S^{1},
$$

т.е. множество $S$ представляет собой квадрат

$$
S=\left\{\left(s_{1}, s_{2}\right): \max \left\{\left|s_{1}\right|,\left|s_{2}\right|\right\}<1\right\} .
$$

Оказывается, что при $s \rightarrow s^{\prime} \in \partial S$ поведение производящей функции $f(s)$ существенно зависит от того, является ли $s^{\prime}$ угловой точкой или нет. Разнообразие в поведении производящей функции вблизи границы области существования приводит к большему разнообразию предельных законов для сопряженных распределений. Изучению таких случаев будет посвящена отдельная работа. 


\section{3. Предельные теоремы для сопряженных распределений.}

3.1. Теорема абелева типа. Условия (А), (В) и (С) являются равномерными аналогами так же обозначенных условий в [19]. Поэтому утверждение теоремы 1.3 из цитируемой работы выполняется равномерно по направлениям $e \in S^{d-1}$.

Имеет смысл привести точную формулировку равномерного аналога этой теоремы.

Рассмотрим семейство функций

$$
f^{(e)}(\tau)=f((h(e)-\sigma(e) \tau) e), \quad e \in S^{d-1},
$$

где $0<\tau<\min _{e \in S^{d-1}} \sigma^{-1}(e) h(e)=\min _{e \in S^{d-1}} a(e)$, a $\sigma(e)=\left\langle\varepsilon^{\prime}(e), e\right\rangle^{-1}$.

Теорема 3.1. Пусть $p(x)$ гамма-подобна с $\alpha>-(d-1) / 2$ и выполнень условия (A), (B) $и$ (C). Тогда при $\tau \downarrow 0$

$$
\sup _{e \in S^{d-1}}\left|\frac{\tau^{\alpha+(d-1) / 2} f^{(e)}(\tau)}{l\left(\tau^{-1}\right)}-c_{\alpha} g(e)\right|=o(1),
$$

где $c_{\alpha}=\Gamma(\alpha+(d-1) / 2)(2 \pi)^{(d-1) / 2}, a$

$$
g(e)=\lambda\left(\varepsilon^{\prime}(e)\right)\left(\lambda_{1}(e) \cdots \lambda_{d-1}(e)\right)^{-1 / 2}=\lambda\left(\varepsilon^{\prime}(e)\right)\left(\operatorname{det} \bar{\Lambda}_{e}^{(0)}\right)^{-1 / 2},
$$

$\bar{\Lambda}_{e}^{(0)}=\operatorname{diag}\left(\lambda_{1}(e), \ldots, \lambda_{d-1}(e)\right)$.

Пусть $e_{\tau} \rightarrow e$ при $\tau \rightarrow 0$. Из теоремы 3.1 следует, что

$$
\left|\frac{\tau^{\alpha+(d-1) / 2} f^{\left(e_{\tau}\right)}(\tau)}{l\left(\tau^{-1}\right)}-c_{\alpha} g(e)\right|=o(1)
$$

при любой скорости приближения $e_{\tau}$ к пределу. Это означает, что нами установлена сильная форма абелевой теоремы, в которой нет ограничений на характер сближения аргумента с границей области существования производящей функции.

Положим для $q>1$

$$
\begin{aligned}
I_{q}(s) & =\int_{\mathbf{R}^{d}}\left(e^{\langle s, x\rangle} p(x)\right)^{q} d x, \\
I_{q}^{(e)}(\tau) & =I_{q}((h(e)-\sigma(e) \tau) e), \quad e \in S^{d-1}, \quad 0<\tau<\min _{e \in S^{d-1}} a(e) .
\end{aligned}
$$

Повторяя с очевидными изменениями рассуждения, приводящие к утверждению теоремы 1.3 в [19] и теоремы 3.1 , можно показать, что при $q(\alpha-1)+(d+1) / 2>0$ и $\tau \downarrow 0$

$$
\sup _{e \in S^{d-1}}\left|\frac{\tau^{q(\alpha-1)+(d+1) / 2} I_{q}^{(e)}(\tau)}{l^{q}\left(\tau^{-1}\right)}-c_{\alpha, q} g_{q}(e)\right|=o(1),
$$

где $c_{\alpha, q}=\Gamma(q(\alpha-1)+(d+1) / 2) q^{-q(\alpha-1)-d}(2 \pi)^{(d-1) / 2}, \quad g_{q}(e)=$ $\lambda^{q}\left(\varepsilon^{\prime}(e)\right)\left(\lambda_{1}(e) \cdots \lambda_{d-1}(e)\right)^{-1 / 2}$. Это предельное соотношение нам понадобится позже. 
Следуя доказательству теоремы 1.3 в [19] и теоремы 3.1 нетрудно также показать, что при $s=(h(e)-\sigma(e) \tau) e, \tau \downarrow 0$, для всех $i=1, \ldots, d$

$$
\sup _{e \in S^{d-1}}\left|\frac{\tau^{\alpha+(d+1) / 2} \partial f(s) / \partial s_{i}}{l\left(\tau^{-1}\right)}-c_{\alpha}^{\prime} g(e) \varepsilon_{i}^{\prime}(e)\right|=o(1)
$$

где $c_{\alpha}^{\prime}=\Gamma(\alpha+(d+1) / 2)(2 \pi)^{(d-1) / 2}$, а $\varepsilon^{\prime}(e)=\left(\varepsilon_{1}^{\prime}(e), \ldots, \varepsilon_{d}^{\prime}(e)\right)^{T}$. Вместе с теоремой 3.1 это дает

$$
\lim _{\tau \rightarrow 0} \sup _{e \in S^{d-1}}\left|\tau \gamma((h(e)-\sigma(e) \tau) e)-\left(\alpha+\frac{d-1}{2}\right) \varepsilon^{\prime}(e)\right|=0,
$$

где, напоминаем, $\gamma(s)=\operatorname{grad} \ln f(s)$ - вектор средних для сопряженного распределения.

3.2. Предельные теоремы для сопряженных распределений. В работах [12], [16], [17] изучались вероятности больших уклонений произвольно высокого порядка для сумм скалярных случайных величин. При этом оказалось, что важно знать поведение сопряженного распределения вблизи границы области изменения параметра $s$. В частности, было установлено, что при соответствующей нормировке сопряженное распределение сходится либо к нормальному, либо к гаммараспределению. Разумеется, это происходит при определенной правильности изменения хвостов исходного распределения.

Здесь мы показываем, что нечто подобное имеет место и в случае многомерных сопряженных распределений. Во всяком случае, гаммаподобные распределения приводят к весьма интересным предельным законам для сопряженных к ним распределений.

Пусть $p^{(s)}(x)$ - плотность сопряженного распределения $\mathbf{P}_{s}$, т.е.

$$
p^{(s)}(x)=\frac{e^{\langle s, x\rangle} p(x)}{f(s)}, \quad x \in \mathbf{R}^{d}, \quad s \in S .
$$

Рассмотрим ортогональное преобразование $C_{e}$, которое переводит вектор $\varepsilon^{(d)}=(0, \ldots, 0,1)^{T}$ в $\varepsilon^{\prime}(e)$, т.е. $C_{e} \varepsilon^{(d)}=\varepsilon^{\prime}(e)$. Положим $p^{(s)}\left(C_{e} x\right)=p_{e}^{(s)}(x)$. Если случайный вектор $\xi$ имеет плотность распределения $p^{(s)}(x)$, то, как легко понять, $p_{e}^{(s)}(x)$ отвечает случайному вектору $C_{e}^{T} \xi$.

Обозначим векторы из первых $d-1$ компонент $x$ через $y$, а последнюю компоненту $x$ обозначим $z$. Таким образом,

$$
x=(y, z), \quad p_{e}^{(s)}(x)=p_{e}^{(s)}(y, z) .
$$

Справедлива следующая теорема.

Теорема 3.2. В условиях теоремь 3.1 при $s=(h(e)-\sigma(e) \tau) e$, $\tau \downarrow 0, u$ ^юбом $\delta>0$

$$
\sup _{e \in S^{d-1}} \sup _{y \in \mathbf{R}^{d-1}, z \geqslant \delta}\left|\tau^{-(d+1) / 2} p_{e}^{(s)}\left(\tau^{-1 / 2} y, \tau^{-1} z\right)-\pi_{e}(y, z)\right|=o(1),
$$


$2 \partial e$

$$
\pi_{e}(y, z)=\frac{z^{\alpha+(d-3) / 2} e^{-z}}{\Gamma(\alpha+(d-1) / 2)} \varphi_{B_{e, z}}(y),
$$

а $\varphi_{B_{e, z}}$ - плотность $(d-1)$-мерного нормального распределения $с$ нулевьим вектором средних и ковариаиионной матрицей $B_{e, z}=z\left(\bar{\Lambda}_{e}^{(0)}\right)^{-1}$.

Прежде чем доказывать сформулированную теорему, сделаем ряд замечаний.

Нетрудно понять, что последняя компонента случайного вектора, имеющего плотность распределения $\pi_{e}(y, z)$, распределена по закону гамма с параметром формы $\alpha+(d-1) / 2$. В то же время условное распределение первых $d-1$ компонент при условии, что последняя принимает значение $z$, является $(d-1)$-мерным нормальным с нулевым вектором средних и ковариационной матрицей $B_{e, z}$. Последнее означает, что маргинальное распределение первых $d-1$ компонент представляет собой смесь нормальных распределений, в которой в качестве параметра смеси выступает масштаб, а весовой функцией является плотность упомянутого гамма-распределения.

Утверждение теоремы допускает простую интерпретацию. Представим случайный вектор $\xi$, имеющий $p^{(s)}(x)$ плотностью своего распределения, в виде

$$
\xi=\xi^{\prime}+\left\langle\xi, \varepsilon^{\prime}(e)\right\rangle \varepsilon^{\prime}(e) .
$$

Из сформулированной теоремы следует, что при $s=(h(e)-\sigma(e) \tau) e$, $\tau \downarrow 0$, составляющая $\left\langle\xi, \varepsilon^{\prime}(e)\right\rangle$ имеет в качестве предельного закона гамма-распределение, тогда как вектор $\xi^{\prime}$ - смесь $(d-1)$-мерных нормальных распределений.

Нетрудно также понять, что нельзя без дополнительных ограничений, налагаемых на $\alpha$, снять в теореме 3.2 ограничения снизу на $z$. Действительно, при $x=0$, согласно теореме 1.3 в [19], имеем

$$
\tau^{-(d+1) / 2} p^{(s)}(0)=\frac{\tau^{-(d+1) / 2} p(0)}{f(s)} \sim \frac{c \tau^{\alpha-1}}{l\left(\tau^{-1}\right)},
$$

если, разумеется, $0<p(0)<\infty$ (ср. с леммой 5 в [17]).

Доказательство теоремы 3.2. Далее на протяжении оставшейся части работы $c$ будет обозначать положительную постоянную, конкретное значение которой для нас неважно, т.е. считается, что $c+c=c, c^{2}=c$ и т.д. Через $\omega(t)$ обозначим произвольную неотрицательную функцию такую, что $\lim _{t \rightarrow \infty} \omega(t)=0$, а через $\theta-$ любую величину, принимающую значения из интервала $[-1,1]$.

Зафиксируем $\delta>0$, после чего запишем

$$
\begin{aligned}
S & =\sup _{e \in S^{d-1}} \sup _{y \in \mathbf{R}^{d-1}, z \geqslant \delta}\left|\tau^{-(d+1) / 2} p_{e}^{(s)}\left(\tau^{-1 / 2} y, \tau^{-1} z\right)-\pi_{e}(y, z)\right| \\
& =\sup _{e \in S^{d-1}} \max \left(S_{1}, S_{2}\right),
\end{aligned}
$$


где

$$
\begin{aligned}
& S_{1}=\sup _{\left(x=(y, z): x \in Y_{12}^{\prime}\right)}\left|\tau^{-(d+1) / 2} p_{e}^{(s)}\left(\tau^{-1 / 2} y, \tau^{-1} z\right)-\pi_{e}(y, z)\right|, \\
& S_{2}=\sup _{\left(x=(y, z): x \notin Y_{12}^{\prime}, z \geqslant \delta\right)}\left|\tau^{-(d+1) / 2} p_{e}^{(s)}\left(\tau^{-1 / 2} y, \tau^{-1} z\right)-\pi_{e}(y, z)\right|,
\end{aligned}
$$

а $Y_{12}^{\prime}=\{x=(y, z):|y| \leqslant M, \delta \leqslant z \leqslant N\}$, где $M$ и $N$ - произвольно большие положительные числа.

Для $x=(y, z) \in Y_{12}=\left\{x:|y| \leqslant M \tau^{-1 / 2}, \delta \leqslant z \tau \leqslant N\right\}$ имеем (см. оценку $f_{12}(s)$ в доказательстве теоремы 1.3 в [19])

$$
\bar{\varepsilon}=z^{-1} y \tau^{1 / 2}+O\left(\tau^{3 / 2}\right), \quad \varepsilon_{d}=1+O(\tau), \quad \sigma(e)=e_{d}^{-1}+O(\tau),
$$

и, следовательно,

$$
\bar{\varepsilon}^{T} \bar{\Lambda}_{e}^{(0)} \bar{\varepsilon}=\tau z^{-2} y^{T} \bar{\Lambda}_{e}^{(0)} y+o(\tau), \quad \tau \sigma(e)\langle e, \varepsilon\rangle=\tau+O\left(\tau^{3 / 2}\right)
$$

где $\bar{\varepsilon}=\left(\varepsilon_{1}, \ldots, \varepsilon_{d-1}\right)^{T}$. Поэтому $\langle s, x\rangle-a(x)=-z-(2 z)^{-1} y^{T} \bar{\Lambda}_{e}^{(0)} y+$ $o(1)$, откуда ввиду теоремы 3.1 и $(2.1)$, где, напоминаем, $\beta=\alpha-1$, получаем $\tau^{-(d+1) / 2} p_{e}^{(s)}\left(\tau^{-1 / 2} y, \tau^{-1} z\right)=\pi_{e}(y, z)+o(1)$ равномерно по $x \in$ $Y_{12}^{\prime}$ и, конечно же, по $e \in S^{d-1}$. Таким образом,

$$
S_{1}=o(1)
$$

равномерно по $e \in S^{d-1}$.

Приступим к оценке $S_{2}$ в (3.3). Легко видеть, что

$$
S_{2} \leqslant \sup _{x \notin Y_{12}^{\prime}, z \geqslant \delta} \pi_{e}(y, z)+\sup _{x \notin Y_{12}^{\prime}, z \geqslant \delta} \tau^{-(d+1) / 2} p_{e}^{(s)}\left(\tau^{-1 / 2} y, \tau^{-1} z\right)=S_{21}+S_{22} .
$$

Далее, из определения $\pi_{e}(y, z)$ следует, что

$$
S_{21} \leqslant c \sup _{z>N}\left(z^{\alpha^{\prime}-1} e^{-z}\right)+c \sup _{z \geqslant \delta}\left(z^{\alpha^{\prime}-1} e^{-z-c M^{2} z^{-1}}\right), \quad \alpha^{\prime}=\alpha+\frac{d-1}{2} .
$$

Поскольку $\delta$ зафиксировано, то

$$
\begin{aligned}
\sup _{z \geqslant \delta}\left(z^{\alpha^{\prime}-1} e^{-z-c M^{2} z^{-1}}\right) & \leqslant \sup _{z \geqslant \delta}\left(z^{\alpha^{\prime}-1} e^{-z / 2}\right) \sup _{z>0}\left(e^{-z / 2-c M^{2} z^{-1}}\right) \\
& =e^{-c M} \sup _{z \geqslant \delta}\left(z^{\alpha^{\prime}-1} e^{-z / 2}\right)=\omega(M) .
\end{aligned}
$$

Таким образом,

$$
S_{21} \leqslant \omega(M)+\omega(N) .
$$

Воспользуемся обозначениями $X_{2}, X_{3}, X_{12}$ и $X_{13}$ из доказательства теоремы 1.3 в [19], поменяв в них только местами $\delta$ и $\delta^{\prime}$. Заметим, что область $Y_{12}^{\prime}$, очевидным образом связанная с $Y_{12}$, отвечает области $X_{12}$ в доказательстве теоремы 1.3 в [19]. Не ограничивая общности, можно считать, что $X_{12} \subset Y_{12}$, т.е. $\mathbf{R}^{d} \backslash Y_{12} \subset \mathbf{R}^{d} \backslash X_{12}$, откуда

$$
S_{22} \leqslant \max \left(S^{\prime}, S^{\prime \prime}, S^{\prime \prime \prime}\right)
$$


где справа стоят супремумы от $\tau^{-(d+1) / 2} p_{e}^{(s)}(y, z)$ по областям $X_{2}^{\prime}, X_{3}^{\prime}$ и $X_{13}$ соответственно, где $X_{i}^{\prime}=X_{i} \cap\left(x: z \geqslant \delta \tau^{-1}\right), i=2,3$. При $x \in X_{2}^{\prime}$ имеем

$$
\langle s, x\rangle-a(x) \leqslant-c M^{2} \tau|x|, \quad b(x) \leqslant c|x|^{\alpha-1} l\left(|x|^{-1}\right)
$$

откуда, согласно (2.1) и теореме 3.1 ,

$$
\begin{aligned}
& \tau^{-(d+1) / 2} p_{e}^{(s)}(x) \leqslant c(\tau|x|)^{\alpha-1} \frac{l(|x|)}{l(1 / \tau)} e^{-c M^{2} \tau|x|} \\
& \quad \leqslant c(\tau|x|)^{\alpha-1} \max \left((\tau|x|)^{\eta},(\tau|x|)^{-\eta}\right) e^{-c M^{2} \tau|x|}=\omega(M), \quad \eta>0 .
\end{aligned}
$$

Напомним, что $\delta$ фиксировано, а $\tau|x| \geqslant \delta$. Таким образом, $S^{\prime} \leqslant \omega(M)$ равномерно по $e \in S^{d-1}$. При $x \in X_{3}^{\prime}$ в соответствии с условием $(C)$ имеем при всех достаточно малых $\tau$

$$
\langle s, x\rangle-a(x) \leqslant-c|x|, \quad b(x) \leqslant c|x|^{\alpha-1} l\left(|x|^{-1}\right) .
$$

С помощью (2.1) и теоремы 3.1 получаем

$$
\tau^{-(d+1) / 2} p_{e}^{(s)}(x) \leqslant c \sup _{u \geqslant \delta}\left(u^{\alpha-1} \max \left(u^{\eta}, u^{-\eta}\right) e^{-(c / \tau) u}\right)=o(1), \quad \eta>0 .
$$

Иными словами, $S^{\prime \prime}=o(1)$ равномерно по $e \in S^{d-1}$. Если же $x \in X_{13}$, то

$$
\tau^{-(d+1) / 2} p_{e}^{(s)}(x) \leqslant c(\tau|x|)^{\alpha-1+\eta} \frac{l(|x|)}{l\left(\tau^{-1}\right)} e^{-c \tau|x|} \leqslant \omega(M), \quad \eta>0 .
$$

Тем самым, для всех достаточно малых $\tau$ получаем

$$
S_{22} \leqslant \omega(\min (M, N))
$$

равномерно по $e \in S^{d-1}$.

Собирая вместе (3.3)-(3.8), получаем утверждение теоремы. Теорема доказана.

Несложные вычисления показывают, что ковариационная матрица, отвечающая $\pi_{e}(y, z)$, имеет вид

$$
\Sigma_{e}=\left(\alpha+\frac{d-1}{2}\right) \operatorname{diag}\left(\lambda_{1}^{-1}(e), \ldots, \lambda_{d-1}^{-1}(e), 1\right) .
$$

Положим

$$
D_{\tau}=\operatorname{diag}\left(\tau^{1 / 2}, \ldots, \tau^{1 / 2}, \tau\right)
$$

Из теоремы 3.2 следует, что случайный вектор $\Sigma_{e}^{-1 / 2} D_{\tau} C_{e}^{T} \xi$ сходится по $\mathbf{P}_{s}$-распределению к случайному вектору с единичной ковариационной матрицей, плотность которого имеет вид

$$
\pi(y, z)=\frac{1}{\sigma} \frac{(z / \sigma)^{\alpha+(d-3) / 2} e^{-z / \sigma}}{\Gamma(\alpha+(d-1) / 2)} \varphi_{\sigma z I}(y), \quad y \in \mathbf{R}^{d-1}, \quad z \in \mathbf{R}_{+}^{1}
$$

где $I$ - единичная матрица, а $\sigma=(\alpha+(d-1) / 2)^{-1 / 2}$.

С другой стороны, случайный вектор $\xi_{s}=B^{-1 / 2}(s) \xi$, где $B(s)$ ковариационная матрица, отвечающая $p^{(s)}(x)$, также имеет единичную 
ковариационную матрицу. Однако пока ничего не известно о сходимости этого вектора. Заметим попутно, что положительно определенная матрица $B(s)$ допускает представление $B(s)=C(s) B_{0}(s) C^{T}(s)$, где ортогональная матрица $C(s)$ и диагональная матрица $B_{0}(s)$ составлены, соответственно, из собственных векторов и соответствующих им собственных чисел. Поскольку матрица $B(s)$ непрерывна в $S$, то матрицы $C(s)$ и $B_{0}(s)$ тоже непрерывны (см., например, $[11$, гл. 7]).

Сходимость по распределению $\xi_{s}$ устанавливается в следующих двух леммах.

Лемма 3.1. Пусть выполнены условия (A), (B) и (C). Тогда при $s=(h(e)-\sigma(e) \tau) e, \tau \downarrow 0$,

$$
\sup _{e \in S^{d-1}}\left\|D_{\tau} C_{e}^{T} B(s) C_{e} D_{\tau}-\Sigma_{e}\right\|=o(1),
$$

где $B(s)$ - ковариачионная матрича, отвечающая $p^{(s)}(x)$, a $\|\cdot\|$ любая из норм матрицьл.

Д ок а з а т ел ь с т в о. Ввиду теоремы 3.2 достаточно установить равномерную интегрируемость $\xi_{s}^{\prime}=D_{\tau} C_{e}^{T} \xi$. Легко видеть, что

$$
\mathbf{E}_{s}\left|\xi_{s}^{\prime}\right|^{2}=\int_{\mathbf{R}^{d-1} \times \mathbf{R}^{1}}\left(\tau|y|^{2}+\tau^{2} z^{2}\right) p_{e}^{(s)}(y, z) d y d z,
$$

или, вспоминая определение $p_{e}^{(s)}(y, z)$,

$$
\mathbf{E}_{s}\left|\xi_{s}^{\prime}\right|^{2}=\frac{1}{f(s)} \int_{\mathbf{R}^{d}}\left(\tau|y|^{2}+\tau^{2} z^{2}\right) p\left(C_{e} \bar{x}\right) e^{\left\langle s, C_{e} \bar{x}\right\rangle} d \bar{x}
$$

где $x=\left(y_{1}, \ldots, y_{d-1}, z\right)=(y, z)$.

Рассмотрим

$$
\bar{f}(s)=\int_{\mathbf{R}^{d}}\left(\tau|y|^{2}+\tau^{2} z^{2}\right) p\left(C_{e} \bar{x}\right) e^{\left\langle s, C_{e} \bar{x}\right\rangle} d \bar{x} .
$$

Пусть области $X_{i}, i=1, \ldots, 4, X_{1 i}, i=1,2,3$, те же, что и в доказательстве теоремы 1.3 в [19]. Обозначим через $\bar{f}_{i}(s), \bar{f}_{1 i}(s)$ части интеграла $\bar{f}(s)$, отвечающие указанным областям. Необходимо показать, что все отношения $\bar{f}_{i}(s) / f(s), \bar{f}_{1 i}(s) / f(s)$, кроме $\bar{f}_{12}(s) / f(s)$, могут быть сделаны произвольно малыми. Заметим, что интегралы $\bar{f}_{i}(s), \bar{f}_{1 i}(s)$ оцениваются почти дословно так же, как соответствующие им интегралы $f_{i}(s), f_{1 i}(s)$. Например, при оценке $\bar{f}_{2}(s)$ следует воспользоваться очевидньтм неравенством

$$
|y|^{2}+\tau x^{2} \leqslant c r^{2}\left(|\bar{\varepsilon}|^{2}+\tau\right), \quad C_{e} x \in X_{2},
$$

где $x=(y, z)=r \varepsilon, \varepsilon=\left(\varepsilon_{1}, \ldots, \varepsilon_{d}\right)^{T}, \vec{\varepsilon}=\left(\varepsilon_{1}, \ldots, \varepsilon_{d-1}\right)^{T}$. Далее,

$$
\begin{aligned}
\bar{f}_{2}(s) \leqslant & c \tau \int_{L}^{\infty} r^{\alpha+d} l(r) e^{-c \tau r}\left(\int_{|\bar{\varepsilon}| \geqslant M \tau^{1 / 2}}|\bar{\varepsilon}|^{2} \exp \left(-c r|\bar{\varepsilon}|^{2}\right) d \bar{\varepsilon}\right) d r \\
& +c \tau^{2} \int_{L}^{\infty} r^{\alpha+d} l(r) e^{-c \tau r}\left(\int_{|\bar{\varepsilon}| \geqslant M \tau^{1 / 2}} \exp \left(-c r|\bar{\varepsilon}|^{2}\right) d \bar{\varepsilon}\right) d r .
\end{aligned}
$$


Рассуждая так же, как и при оценке $f_{2}(s)$ в доказательстве теоремы 1.3 в [19], получаем $\bar{f}_{2}(s) \leqslant \tau^{-\alpha-(d-1) / 2} l\left(\tau^{-1}\right) \omega(M)$, откуда ввиду теоремы 3.1 $\bar{f}_{2}(s) / f(s) \leqslant \omega(M)$. Столь же просто оцениваются и остальные соотношения. Лемма доказана.

Пусть $\mathscr{C}_{0}$ объединяет все ортогональные $d \times d$-матрицы, имеющие последнюю строку, а следовательно, и столбец, вида $(0, \ldots, 0, a)$ с $|a|=1$. Ясно, что плотность $\pi(y, z)$ инвариантна относительно преобразований $C \in \mathscr{C}_{0}$.

Лемма 3.2. Положим $\bar{p}^{(s)}(x)=(\operatorname{det} B(s))^{1 / 2} p^{(s)}\left(B^{1 / 2}(s) x\right)$. В условиях теоремы 3.2

$$
\lim _{s \rightarrow \partial S} \sup _{x_{d} \geqslant \delta}\left|\bar{p}^{(s)}(x)-\pi(x)\right|=0 .
$$

Д ок а з а т ел ь с т в о. Пусть $\tau=\tau(s)=\left(h\left(e_{s}\right)-|s|\right) / \sigma\left(e_{s}\right)$. Из леммы 3.1 следует, что

$$
C_{e}^{T} B(s) C_{e}=\tau^{-1} \Sigma(s),
$$

где

$$
\Sigma(s)=\left(\begin{array}{clcc}
\sigma_{1}+o(1) & \ldots & o(1) & o\left(\tau^{-1 / 2}\right) \\
\ldots & \ldots & \ldots & \ldots \\
o(1) & \ldots & \sigma_{d-1}+o(1) & o\left(\tau^{-1 / 2}\right) \\
o\left(\tau^{-1 / 2}\right) & \ldots & o\left(\tau^{-1 / 2}\right) & \sigma_{d} \tau^{-1}+o\left(\tau^{-1}\right)
\end{array}\right)
$$

a $\sigma_{j}, j=1, \ldots, d$, - элементы диагональной матрицы $\Sigma_{e}$. Непосредственной проверкой убеждаемся, что собственные значения матрицы $B(s)$ ведут себя следующим образом:

$$
\left\{\begin{array}{l}
\beta_{j}(s)=\sigma_{j} \tau^{-1}(1+o(1)), \quad j=1, \ldots, d-1, \\
\beta_{d}(s)=\sigma_{d} \tau^{-2}(1+o(1)) .
\end{array}\right.
$$

С другой стороны, как уже отмечалось, $B(s)=C(s) B_{0}(s) C^{T}(s)$, где $B_{0}(s)=\operatorname{diag}\left(\beta_{1}(s), \ldots, \beta_{d}(s)\right)$, а ортогональная матрица $C(s)$ составлена из собственных векторов матрицы $B(s)$. Из леммы 3.1 следует, что

$$
\begin{cases}\tau\left(c_{i 1} c_{j 1} \beta_{1}+\cdots+c_{i d} c_{j d} \beta_{d}\right)=\sigma_{i} \delta_{i j}+o(1), & i, j=1, \ldots, d-1, \\ \tau^{3 / 2}\left(c_{i 1} c_{d 1} \beta_{1}+\cdots+c_{i d} c_{d d} \beta_{d}\right)=o(1), & i=1, \ldots, d-1, \\ \tau^{2}\left(c_{d 1}^{2} \beta_{1}+\cdots+c_{d d}^{2} \beta_{d}\right)=\sigma_{d}+o(1), & \end{cases}
$$

где $\beta_{j}=\beta_{j}(s)$, а $c_{i j}=c_{i j}(s), i, j=1, \ldots, d$, - элементы матрицы $C_{e}^{T} C(s)$.

Из (3.10) и (3.11) следует, что

$$
c_{d d}^{2}=1+o(1), \quad c_{i d}=o\left(\tau^{1 / 2}\right), \quad i=1, \ldots, d-1,
$$

и, значит, $c_{d j}=o\left(\tau^{1 / 2}\right), j=1, \ldots, d-1$. Таким образом,

$$
\lim _{s \rightarrow \partial S} \min _{C \in \mathscr{C}_{0}}\left\|C_{e}^{T} C(s)-C\right\|=0 .
$$


Положим

$$
B^{1 / 2}(s)=C(s) B_{0}^{1 / 2}(s), \quad R(s)=\Sigma_{e}^{-1 / 2} D_{\tau} C_{e}^{T} .
$$

Пусть при $s=s_{k}=\left(h\left(e_{k}\right)-\sigma\left(e_{k}\right) \tau_{k}\right) e_{k}$ последовательности $R\left(s_{k}\right) B^{1 / 2}\left(s_{k}\right)$ и $C_{e_{k}}^{T} C\left(s_{k}\right)$ сходятся, соответственно, к $C^{\prime}$ и $C^{\prime \prime}$. Ввиду $(3.12)$ имеем $C^{\prime \prime} \in$ $\mathscr{C}_{0}$. Тогда $C^{\prime}=\lim _{k \rightarrow \infty} \Sigma_{e_{k}}^{-1 / 2} D_{\tau_{k}} C^{\prime \prime} B_{0}^{1 / 2}\left(s_{k}\right)$, и, следовательно, $C^{\prime} \in \mathscr{C}_{0}$. Далее ввиду леммы 3.1 при $s \rightarrow \partial S$ имеем $(\operatorname{det} B(s))^{1 / 2} \operatorname{det} R(s) \longrightarrow 1$. Но тогда

$$
\begin{aligned}
\sup _{x_{d} \geqslant \delta>0} & \left|(\operatorname{det} B(s))^{1 / 2} p^{(s)}\left(B^{1 / 2}(s) x\right)-\pi(x)\right| \\
\leqslant \sup _{x_{d} \geqslant \delta>0} \mid(\operatorname{det} B(s))^{1 / 2} p^{(s)}\left(R^{-1}(s) R(s) B^{1 / 2}(s) x\right) & -(\operatorname{det} B(s))^{1 / 2} \operatorname{det} R(s) \pi\left(R(s) B^{1 / 2}(s) x\right) \mid \\
& \quad\left[\sup _{x_{d} \geqslant \delta>0}\left|(\operatorname{det} B(s))^{1 / 2} \operatorname{det} R(s) \pi\left(R(s) B^{1 / 2}(s) x\right)-\pi(x)\right|=o(1) .\right.
\end{aligned}
$$

Лемма доказана.

Теорема 3.3. Пусть выполнень условия (A),(B) и (C). Тогда при $n \rightarrow \infty$

$\sup _{s \in S} \sup _{u \in \mathbf{R}^{d}}\left|(2 \pi n)^{d / 2}(\operatorname{det} B(s))^{1 / 2} p_{n s}\left(n^{1 / 2} B^{1 / 2}(s) u+n \gamma(s)\right)-e^{-|u|^{2} / 2}\right|=o(1)$.

Д о к а з а т е л с т в о. Рассмотрим характеристическую функцию

$$
\psi_{s}(t)=\int_{\mathbf{R}^{d}} e^{\imath\langle t, x\rangle} \bar{p}^{(s)}(x) d x, \quad t \in \mathbf{R}^{d},
$$

отвечающую плотности $\bar{p}^{(s)}(x)$.

Из леммы 3.2 немедленно получаем (ср. с (17)-(19) в [17]) следующие соотношения: при $n \rightarrow \infty$ для любого $T>0$

$$
\sup _{s \in S} \sup _{|t|<T}\left|\psi_{s}^{n}\left(\frac{t}{\sqrt{n}}\right)-\exp \left(-\frac{1}{2}|t|^{2}\right)\right|=o(1) ;
$$

для всех достаточно малых $\delta>0$ существует $c_{\delta}>0$ такое, что

$$
\sup _{s \in S}\left|\psi_{s}(t)\right| \leqslant 1-c_{\delta}|t|^{2}, \quad 0<|t| \leqslant \delta
$$

для любого $\delta>0$

$$
\sup _{s \in S} \sup _{|t|>\delta}\left|\psi_{s}(t)\right|=\rho_{\delta}<1
$$

Остается показать, что существует $n_{0}>1$ такое, что $\left|\psi_{s}(t)\right|^{n_{0}}$ интегрируема равномерно по $s \in S$. Представим $s$, как и прежде, в виде

$$
s=(h(e)-\sigma(e) \tau) e, \quad 0<\tau<\min _{e \in S^{d-1}} a(e) .
$$


Из (2.1) имеем

$$
p^{(s)}(x)=\frac{b(x) \exp \left\{-|x|\left(\Delta_{e}\left(e_{x}\right)+\tau \sigma(e)\left\langle e, e_{x}\right\rangle\right)\right\}}{f((h(e)-\tau \sigma(e)) e))} .
$$

Из условий $(\mathrm{A})$ и $(\mathrm{C})$ следует, что $\sup _{e \in S^{d-1}} \sup _{\tau>\tau_{0}} \sup _{x} \bar{p}^{(s)}(x)<\infty$ для любого $\tau_{0}>0$. Это означает, что для любого $\tau_{0}>0$

$$
\sup _{e \in S^{d-1}} \sup _{\tau>\tau_{0}} \int_{\mathbf{R}^{d}}\left|\psi_{s}(t)\right|^{2} d t<\infty
$$

Далее,

$$
\int_{\mathbf{R}^{i}}\left(\bar{p}^{(s)}(x)\right)^{q} d x=(\operatorname{det} B(s))^{(q-1) / 2}(f(s))^{-q} I_{q}(s),
$$

где $I_{q}(s)$ то же, что и в $(3.1)$, а

$$
1<q< \begin{cases}\min \left(2, \frac{d+1}{2(1-\alpha)}\right), & \alpha<1, \\ 2, & \alpha \geqslant 1 .\end{cases}
$$

Из теоремы 3.1, а также из (3.1) и (3.10) следует, что существует конечный предел $\lim _{s \rightarrow \partial S} \int_{\mathbf{R}^{d}}\left(\bar{p}^{(s)}(x)\right)^{q} d x$. Последнее в свою очередь означает (см., например, [5, с. 199])

$$
\limsup _{s \rightarrow \partial S} \int_{\mathbf{R}^{d}}\left|\psi_{s}(t)\right|^{q /(q-1)} d t<\infty .
$$

Таким образом, при $n_{0} \geqslant q /(q-1)$

$$
\sup _{s \in S} \int_{\mathbf{R}^{d}}\left|\psi_{s}(t)\right|^{n_{0}} d t<\infty .
$$

Доказательство теоремы следует теперь из соотношений (3.13)-(3.17) (ср., например, с [9, гл. 4, § 3]). Теорема доказана.

\section{4. Доказательство теоремы 2.1 и ее статистическая интер-} претация.

4.1. Доказательство теоремы 2.1. Рассмотрим хорошо известное тождество

$$
p_{n}(n x)=f^{n}(s) e^{-n\langle s, x\rangle} p_{n s}(n x), \quad s \in S,
$$

где, напомним, $p_{n}(x)$ и $p_{n s}(x)$ - плотности распределения суммы $S_{n}$ относительно $\mathbf{P}_{0}$ и $\mathbf{P}_{s}$ соответственно.

При $x \in X, x=\gamma(s)$, получаем $p_{n}(n x)=\rho^{n}(x) p_{n s}(n \gamma(s))$. Из теоремы 3.3 следует, что при $n \rightarrow \infty$

$$
\sup _{x \in X}\left|\frac{p_{n}(n x)}{\psi_{n}(x) \rho^{n}(x)}-1\right|=o(1) .
$$

Покажем, что $X=\mathbf{R}^{d}$. Поскольку $S$ открыто, то и $X$ открыто. Пусть $X \neq \mathbf{R}^{d}$, т.е. существует $x^{*} \notin X, x^{*} \in \partial X,\left|x^{*}\right|<\infty$. Возьмем произвольную последовательность $\left\{x^{(k)}\right\}_{k=1}^{\infty}, x^{(k)} \in X$, такую, что 
$x^{(k)} \rightarrow x^{*}, k \rightarrow \infty$. Пусть $s^{(k)} \in S$ таковы, что $\gamma\left(s^{(k)}\right)=x^{(k)}$. Поскольку замыкание $S$ компактно, то из последовательности $\left\{s^{(k)}\right\}_{k=1}^{\infty}$ можно выделить сходяшуюся подпоследовательность $\left\{s^{\left(k_{n}\right)}\right\}_{n=1}^{\infty}$. Пусть $s^{*}-$ предел этой последовательности. Поскольку $x^{*}$ лежит на границе $X$, то $s^{*} \in \partial S$. Но тогда, ввиду (3.2), вопреки нашему предположению должно быть $\left|\gamma\left(s^{*}\right)\right|=\left|x^{*}\right|=\infty$. Таким образом, действительно, $X=\mathbf{R}^{d}$, и, следовательно, имеет место (1.2).

4.2. Статистическая интерпретация теоремы 2.1. С точки зрения математической статистики, семейство сопряженных распределений может рассматриваться как экспоненциальное. При этом $s \in S$ выступает в качестве оцениваемого параметра. Независимые случайные векторы $\xi^{(j)}, j=1, \ldots, n$, образуют выборку объема $n$. Отвечающая ей функция правдоподобия имеет вид $\boldsymbol{p}_{n}\left(\xi^{(1)}, \ldots, \xi^{(n)} ; s\right)=\prod_{j=1}^{n} p\left(\xi^{(j)} ; s\right)$, где $p(x ; s)=e^{\langle s, x\rangle} p(x) / f(s)$, или

$$
\begin{aligned}
\boldsymbol{p}_{n}\left(\xi^{(1)}, \ldots, \xi^{(n)} ; s\right) & =f^{-n}(s) \exp \left(\sum_{j=1}^{n}\left\langle s, \xi^{(j)}\right\rangle\right) \prod_{j=1}^{n} p\left(\xi^{(j)}\right) \\
& =f^{-n}(s) \exp (n\langle s, \bar{\xi}\rangle) \prod_{j=1}^{n} p\left(\xi^{(j)}\right)
\end{aligned}
$$

где $\bar{\xi}=n^{-1} \sum_{j=1}^{n} \xi^{(j)}$. Согласно критерию факторизации (см., например, теорему 1.1 .1 в [10]), статистика $\bar{\xi}$ достаточна для параметра $s$. При этом информационная матрица Фишера, отвечаюшая единичному наблюдению, совпадает с ковариационной матрицей $B(s)$.

В качестве оценки максимального правдоподобия выступает статистика $s^{*}=s(\bar{\xi})$, где, напоминаем, $s(x)$ - вектор-функция, обратная $\gamma(s)=\operatorname{grad} \ln f(s)$.

Пусть $S_{\delta}$ - замкнутое подмножество $\operatorname{int} S$, определяемое следующим образом:

$$
S_{\delta}=\{s: s=r e, 0 \leqslant r \leqslant h(e)-\delta\}, \quad \delta>0 .
$$

Легко проверить, что выполнены все условия так называемой локальной асимптотической нормальности (см., например, теорему 2.1 .1 в [10]). Это означает, что логарифм отношения правдоподобия в близких точках вида $s$ и $s+n^{-1 / 2} B^{-1 / 2}(s) t, t \in \mathbf{R}^{d}$, допускает представление

$$
\begin{aligned}
\ln \frac{\boldsymbol{p}\left(\cdot ; s+n^{-1 / 2} B^{-1 / 2}(s) t\right)}{\boldsymbol{p}(\cdot ; s)}= & n^{-1 / 2} \sum_{j=1}^{n}\left\langle B^{-1 / 2}(s)\left(\xi^{(j)}-\gamma(s)\right), t\right\rangle \\
& -\frac{1}{2} t^{T} t+\Delta_{n}(s, t),
\end{aligned}
$$

где функция $\Delta_{n}(s, t)$, в отличие от остаточного члена в теореме 2.1.1 [10], неслучайна и

$$
\lim _{n \rightarrow \infty} \sup _{|t|<T} \sup _{s \in S_{\delta}}\left|\Delta_{n}(s, t)\right|=0 .
$$


Представление (4.1) справедливо для любой плотности, удовлетворяющей условию Крамера с областью $S$. Возникает вопрос: нельзя ли распространить (4.1) на все множество $S$, по крайней мере, для случая гамма-подобных исходных распределений? Теоремы 3.1 и 3.2 позволяют утвердительно ответить на этот вопрос. Не приводя деталей доказательства, отметим следующие существенные моменты.

Пусть $\widehat{s}=\arg \min _{s^{\prime} \in \partial S}\left|s-s^{\prime}\right|$ для $s \in \operatorname{int} S$. Из теоремы 3.1 можно вывести, что при $s \rightarrow \partial S$

$$
|\widehat{s}-s| \asymp\left\|B^{-1 / 2}(s)\right\|
$$

т.е. при любом $T$ и всех достаточно больших $n$ точки $s+n^{-1 / 2} B^{-1 / 2}(s) t$, $|t| \leqslant T$, лежат в $S$. Более того, при $s \rightarrow \partial S, s^{\prime}=s+o(|\widehat{s}-s|)$,

$$
B^{-1 / 2}(s) B\left(s^{\prime}\right) B^{-1 / 2}(s)=I+o(1) \text {. }
$$

Тогда (4.1) имеет место с $\Delta_{n}(s, t)$, удовлетворяющей соотношению

$$
\lim _{n \rightarrow \infty} \sup _{|t|<T} \sup _{s \in S}\left|\Delta_{n}(s, t)\right|=0 \text {. }
$$

Тем самым, получается любопытный пример локальной асимптотической нормальности, равномерной по открытому параметрическому множеству.

\section{СПИСОК ЛИТЕРАТУРЫ}

1. Алешкявичене A. K. Многомерные интегральные предельные теоремы для вероятностей больших уклонений. - Теория вероятн. и ее примен., 1983 , т. 28 , в. 1 , c. $62-82$.

2. von Bahr B. Multi-dimensional integral limit theorems for large deviations. - Ark. Mat., 1967, v. 7, p. 89-99.

3. Боровков А. А., Рогозин Б. А. О центральной предельной теореме в многомерном случае. - Теория вероятн. и ее примен., 1965, т. 10, в. 1, с. 61-69.

4. Боровков A.A., Могульский $A . A$. Большие уклонения и проверка статистических гипотез. I: Большие уклонения сумм случайных векторов. - Труды Ин-та математики СО РАН, 1992, т. 3, с. 1-63.

5. Бхаттачария Р. Н., Ранга Рао Р. Аппроксимация нормальным распределением и асимптотические разложения. М.: Наука, 1982, $288 \mathrm{c.}$

6. Dembo A., Zeitouni O. Large Deviations Techniques and Applications. Boston: Jones and Bartlett, 1993, $346 \mathrm{p}$.

7. Deuschel J.-D., Stroock D. W. Large Deviations. Boston: Academic Press, 1989, 307 p.

8. Ellis R. J. Entropy, Large Deviations and Statistical Mechanics. New York: SpringerVerlag, 1985, $364 \mathrm{p}$.

9. Ибрагимов И.А., Линник Ю.В. Независимые и стационарно связанные величины. М.: Наука, 1965, 524 с.

10. Ибрагимов И.А., Хасьминский Р. З. Асимптотическая теория оценивания. М.: Наука, 1979, 527 с.

11. Ланкастер П. Теория матриц. М.: Наука, 1978, 280 с.

12. Нагаев $A . B$. Большие уклонения для одного класса распределений. - Предельные теоремы теории вероятностей. Ташкент: Изд-во АН УзССР, 1963, с. 55-68. 
13. Нагаев A.B. Предельные теоремы для сумм независимых двумерных спучайных векторов. - Предельные теоремы и статистические выводы. Ташкент: Фан, 1966, с. 83-89.

14. Нагаев $A . B$. Локальные предельные теоремы специального вида, учитывающие большие уклонения. - Изв. АН УзССР, сер. физ.-матем., 1970, т. 14, в. 1, с. 29-34.

15. Нагаев A.В., Сакоян С.К. Предельные теоремы, учитываюшие большие уклонения, в $\mathbf{R}^{k}$. - Докл. АН СССР, 1972, т. 204, в. 3, с. 554-556.

16. Нагаев A.B., Ходжабагян C. C. Предельные теоремы, учитываюшие большие уклонения для сумм положительных случайных величин. - Литов. матем. сб., 1974, т. 14 , в. 1 , с. $149-163$.

17. Нагаев $A . B$. Большие уклонения для сумм решетчатых случайных величин при выполнении условия Крамера. - Дискретн. матем., 1998, т. 10, в. 3, с. 115-130.

18. Нагаев А.В. Крамеровские большие уклонения в случае, когда крайнее сопряженное распределение имеет тяжелый хвост. - Теория вероятн. и ее примен., 1998 , т. 43 , в. 3, с. $456-475$.

19. Nagaev A.V., Zaigraev A. Abelian theorems for a class of probability distributions in $\mathbf{R}^{d}$ and their application. - J. Math. Sci. (New York), 2000, v. 99, № 4, p. 14541462.

20. Нагаев С. В. Некоторые предельные теоремы для больших уклонений. - Теория вероятн. и ее примен., 1965, т. 10, в. 2, с. 231-254.

21. Nagaev $S$. $V$. Large deviations of sums of independent random variables. - Ann. Probab., 1979, v. 7, № 5, p. 745-789.

22. Ney $P$. Dominating points and the asymptotics of large deviations for random walk on $\mathbf{R}^{d}$. - Ann. Probab., 1983, v. 11, № 1, p. 158-167.

23. Osipov $L$. V. On large deviations for sums of random vectors in $\mathbf{R}^{k}$. - J. Multivariate Anal., 1981, v. 11, № 2, p. 115-126.

24. Петров B. В. О вероятностях больших уклонений сумм независимых случайных величин. - Теория вероятн. и ее примен., 1965, т. 10, в. 2, с. 310-322.

25. Resnick S.I. Extreme Values, Regular Variation, and Point Processes. New York: Springer-Verlag, 1987, $320 \mathrm{p}$.

26. Рихтер B. Локальные предельные теоремы для больших уклонений. - Докл. АН CCCP, 1957, т. 115, с. 53-56.

27. Rozovsky L. V. On probabilities of large deviations in some classes of $k$-dimensional Borel sets. - J. Multivariate Anal., 1985, v. 17, № 1, p. 1-26.

28. Розовский Л. В. Вероятности больших уклонений на всей оси. - Теория вероятн. и ее примен., 1993, т. 38, в. 1, с. 79-109.

29. Розовский Л.В. Вероятности больших уклонений суммы независимых случайных величин с обшей функцией распределения из области притяжения несимметричного устойчивого закона. - Теория вероятн. и ее примен., 1997, т. 42, в. 3, c. $496-530$.

30. Рокафеллар Р. Т. Выпуклый анализ. М.: Мир, 1973, 468 с.

31. Саулис Л., Статулявичюс В. Предельные теоремы о больших уклонениях. Вильнюс: Мокслас, 1989, 208 с.

32. Светулявичене B.K. О вероятностях больших уклонений для сумм случайных векторов. - Литов. матем. сб., 1981, т. 21, в. 2, с. 191-199.

Поступила в редакцию 4.IV.2000

Исправленный вариант 23.I. 2001 